\title{
Nonmonotonic saturation characteristic and excited state absorption in long optical fiber
}

\author{
P. Stremplewski • C. Koepke · D. Piatkowski • \\ M. Rozanski
}

Received: 21 May 2010 / Revised version: 1 July 2010 / Published online: 15 September 2010

(c) The Author(s) 2010. This article is published with open access at Springerlink.com

\begin{abstract}
A sort of nontrivial competition between the excited state absorption (ESA) and optical gain is shown and interpreted for $9 \mathrm{~m}$-long optical fiber. Using a typical $\mathrm{SiO}_{2}: \mathrm{Nd}^{3+}$ fiber we observe an interesting nonmonotonic dependence of the gain on signal (probe beam) intensity with substantial contribution of the ESA. This behavior is simulated via kinetic analysis.
\end{abstract}

\section{Introduction}

Examinations aimed at more efficient broadband fiber amplifiers seem still to be a hot subject [1-4]. The key characteristics to evaluate amplifying materials are the optical gain (usually expressed in $\mathrm{dB}$ when measured at a given wavelength) [5] and/or the excited state absorption/gain spectra with ground state absorption bleaching whenever it occurs $[6,7]$. When dealing with bulk samples the problem of spectroscopic characteristics of the excited state is rather well recognized [8-10]. However, in the case of long fiber some new phenomena appear. One of them is decay of the excitation strength along the fiber, others are various saturation effects in the long active medium [11]. At first glance some of these effects seem to be in the long fiber virtually the same as for the bulk sample but with a much higher concentration of dopant. This would be because of the multiplying action of the long active medium. In the present work we focus our attention on such kind of effect, namely on unusual sensitivity of the ESA/gain balance to the signal level in the long

P. Stremplewski $(\varangle) \cdot$ C. Koepke · D. Piatkowski · M. Rozanski Institute of Physics, Nicolaus Copernicus University, Grudziądzka 5, 87-100 Toruń, Poland

e-mail: patstrem@fizyka.umk.pl fiber. It appears that there is a competition between the ESA and gain which depends on the fiber length and which does not calibrate to the case of bulk sample with suitably higher dopant concentration.

In a theoretical description of fiber amplifiers the ESA is treated usually as "pump ESA" or "signal ESA" i.e. only in the context of direct losses of populations or just reabsorption caused by transitions at exactly the same wavelengths as pump or signal [12]. However, when one needs to amplify a broadband signal, the ESA occurring at wavelength different from the amplified signal can still play a crucial role in the process.

\section{Experimental setup}

The measurements of the excited state characteristics (ESC) have been performed using pump and probe method with double modulation and two lock-in amplifiers as shown in Fig. 1. The used setup is virtually the same as reported in [13]. Pump beam is provided by an Ar laser operating at $514 \mathrm{~nm}, 0.6 \mathrm{~W}$. Probe beam is provided by a tungstenhalogen lamp $(250 \mathrm{~W})$ and its intensity is controlled by a diaphragm. Pumping beam is modulated at frequency $5 \mathrm{~Hz}$ whereas the probe beam is modulated at frequency $1 \mathrm{kHz}$. Detailed account of the system is given in $[7,10]$.

Laser beam creates population of ions in the excited state, ${ }^{4} \mathrm{~F}_{3 / 2}$, and absorption occurs from this state and from the ground state ${ }^{4} \mathrm{I}_{9 / 2}$. The first lock-in registers the signal of the photodiode PD at the input and operates at the frequency of the probe beam modulation. The second lock-in amplifier registers the output signal of the first lock-in at its input and operates at the frequency of the pump beam modulation, hence its output is proportional to the difference between 
Fig. 1 Experimental setup for the ESC spectra measurements in fibers

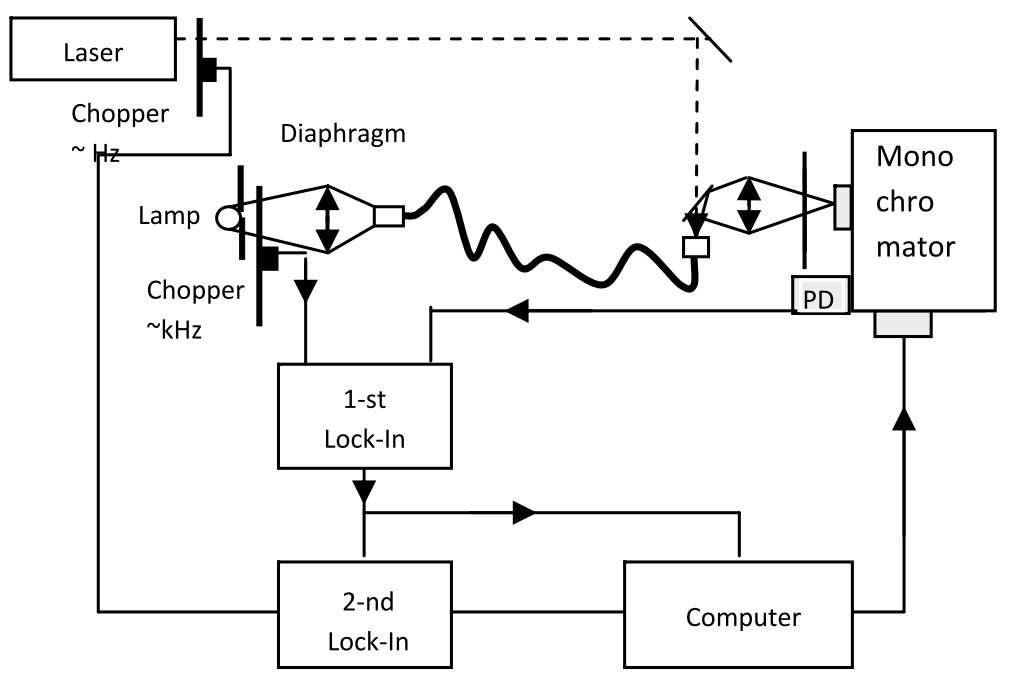

signals in the presence and absence of the pump beam. The output signal of the second amplifier, divided by the probe beam intensity, leads to the ESC spectrum which can be described by the following formula $[7,13]$ :

$$
\begin{aligned}
\operatorname{ESC}(\lambda) & \equiv \frac{\Delta I(\lambda)}{I(\lambda)} A \\
& =A l\left(N^{*} \sigma_{\mathrm{GSA}}(\lambda)+\sum_{i} N_{i}^{*}\left(\sigma_{\mathrm{SE}_{i}}(\lambda)-\sigma_{\mathrm{ESA}_{i}}(\lambda)\right)\right)
\end{aligned}
$$

where $\Delta I(\lambda)$ is the difference between transmitted signal in the presence $\left(I_{p}\right)$ and in the absence $\left(I_{u}\right)$ of the pump beam; the output signal of the 2-nd lock-in, $I(\lambda)$, is the signal transmitted in the absence of the pump beam, $\sigma_{\mathrm{GSA}}(\lambda), \sigma_{\mathrm{SE}_{i}}(\lambda), \sigma_{\mathrm{ESA}_{i}}(\lambda)$ are ground state absorption, stimulated emission and exited state absorption cross sections respectively, $N_{i}^{*}$ is the occupation of $i$ th excited state, $N^{*}$ is total occupation of the excited states, $A$ is amplification factor of the 2-nd lock-in and $l$ is the sample length. Note that the ESC spectrum consists of the SE, ESA and GSA (bleaching) features. Equation (1) is approximated (it has no exponential character) but in the condition of experiment, with relatively small fraction of the excited ions $\left(N^{*}\right)$, such an approximation works very well.

The sample was a 9 meter $\mathrm{SiO}_{2}$ double core fiber doped with $\mathrm{Nd}^{3+}$ ions. The doped core is of $13.6 \mu \mathrm{m}$ diameter and it is placed inside of the second, D is the shaped core (cladding), of $447 \mu \mathrm{m}$ diameter, created to make pumping of the activated core more efficient. The dopant concentration in the sample was $2500 \mathrm{ppm}$. The sample has been produced and provided by the Department of Optical Fibers Technology, University of Maria Curie-Skłodowska, Lublin, Poland.

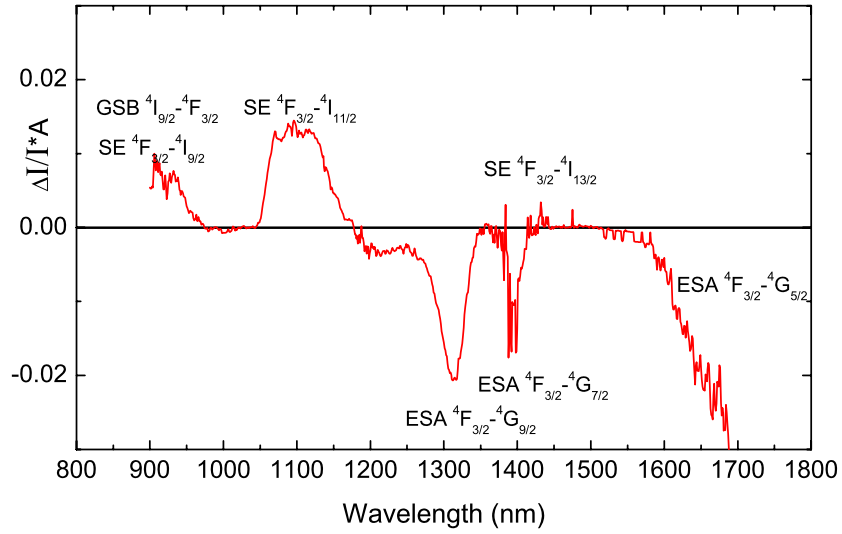

Fig. 2 ESC spectrum for 9 meters $\mathrm{Nd}^{3+}$ doped fiber

\section{Experimental results}

The exemplary ESA spectrum is shown in Fig. 2. In this figure the ESA peaks are negative, SE and bleaching signals are positive \{see definition (1) \}. In this spectrum we can see stimulated emission transitions: ${ }^{4} \mathrm{~F}_{3 / 2} \rightarrow{ }^{4} \mathrm{I}_{9 / 2}(911 \mathrm{~nm})$, ${ }^{4} \mathrm{~F}_{3 / 2} \rightarrow{ }^{4} \mathrm{I}_{11 / 2}(1097 \mathrm{~nm})$, additionally we expect another emission ${ }^{4} \mathrm{~F}_{3 / 2} \rightarrow{ }^{4} \mathrm{I}_{13 / 2}(1385 \mathrm{~nm})$, but in the same region there is the ESA transition ${ }^{4} \mathrm{~F}_{3 / 2} \rightarrow{ }^{4} \mathrm{G}_{7 / 2}$ whose signal is stronger and makes this part of spectrum negative. On the negative side of the spectrum there are the ESA signals: ${ }^{4} \mathrm{~F}_{3 / 2} \rightarrow{ }^{4} \mathrm{G}_{9 / 2}(1306 \mathrm{~nm})$ and ${ }^{4} \mathrm{~F}_{3 / 2} \rightarrow{ }^{4} \mathrm{G}_{5 / 2}(1650 \mathrm{~nm})$. In this figure we can also find bleaching signal: ${ }^{4} \mathrm{I}_{9 / 2} \rightarrow{ }^{4} \mathrm{~F}_{3 / 2}$ (933 nm).

We have also performed the ESC spectra measurements but with different probe beam intensity. Thus we have obtained different spectra and the results of first testing measurements are shown in Fig. 3.

When comparing with the data obtained for bulk glass sample with $\mathrm{Nd}^{3+}$ ions [14] here the relatively broad peaks of weakly defined maxima can indicate saturation. 


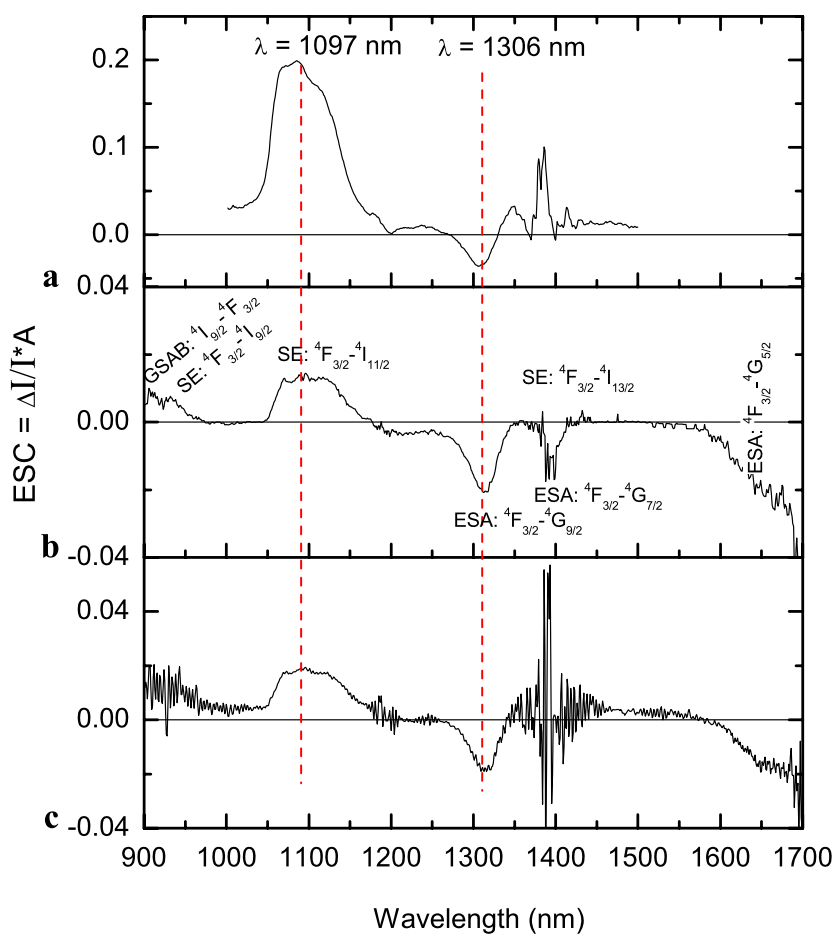

Fig. 3 Comparison of the ESC spectra measured with different intensities of the probe beam $I$, low $I(\mathbf{a})$, intermediate I (b) and high I (c)

The spectrum on the top has been measured using a low intensity of the probe beam, and the spectra below are results of measurements with higher and higher intensities of the probe beam. The growing noise with signal intensity $I$ seen in Fig. 3(a)-(c) is connected with experimental conditions. When increasing signal intensity $I$, to avoid saturation of the photodiode detector and maintain all the electronics parameters constant, we compensated the $I$ increase at the sample output (and input of the monochromator) by appropriate decrease of the slit aperture. Thus with decreasing monochromator slit the signal/noise ratio became worse. Hence we can see a growing noise level of $\Delta I / I$ spectra going from (a) to (c). Moreover, around $1385 \mathrm{~nm}$ in our glass a well-known absorption peak of the $\mathrm{OH}^{-}$groups appears, which makes our sample less transparent in this region when measuring the $\Delta I(\lambda)$ and $I(\lambda)$ spectra. As a result, after division, $\Delta I / I$, a huge noise is obtained at $\sim 1385 \mathrm{~nm}$ in Fig. 3(c). In spite of these drawbacks we can clearly see a decrease of the signal $\Delta I / I$ at the wavelength $1097 \mathrm{~nm}$ when the intensity of the probe beam increases, whereas the signal at $1306 \mathrm{~nm}$ remains almost the same. We can also observe an interesting change of the signal at $1385 \mathrm{~nm}$; when intensity of the probe beam is small, the signal is positive, so we see stimulated emission ${ }^{4} \mathrm{~F}_{3 / 2} \rightarrow{ }^{4} \mathrm{I}_{13 / 2}$ but when we use higher intensity of the probe beam, the signal is negative and now we are able to see ESA transition ${ }^{4} \mathrm{~F}_{3 / 2} \rightarrow{ }^{4} \mathrm{G}_{7 / 2}$. Thus one can separate signals of the ESA and SE despite of their overlap at the same wavelengths. When the probe

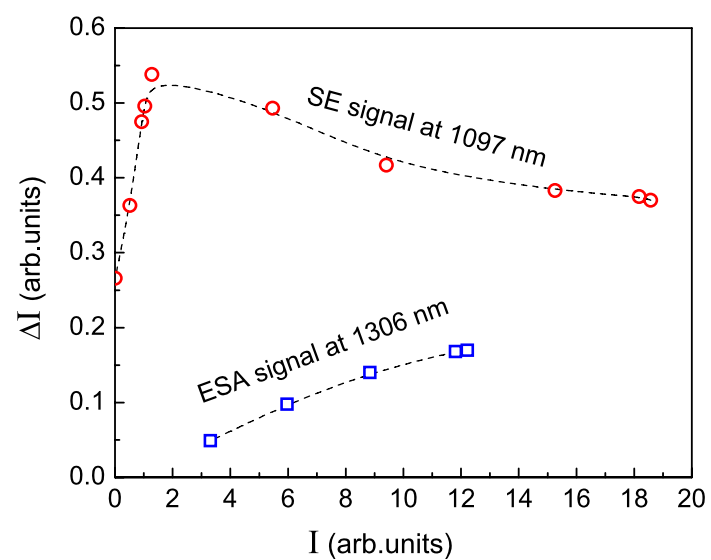

Fig. $4 \Delta I$ signals responsible for SE transitions (on the top) and ESA transitions (below) as a function of probe beam intensity. Dashed lines are eye guides only

beam intensity increases, signal of stimulated emission goes down.

Because of such a strange behavior of the long sample we decided to test more systematically the interdependence between the beam intensity of the probe and signals at two points on the spectrum. We measured signals at the output of the second lock-in $(\Delta I)$ at $1097 \mathrm{~nm}\left(\mathrm{SE}:{ }^{4} \mathrm{~F}_{3 / 2} \rightarrow{ }^{4} \mathrm{I}_{11 / 2}\right)$ and at $1306 \mathrm{~nm}\left(\mathrm{ESA}:{ }^{4} \mathrm{~F}_{3 / 2} \rightarrow{ }^{4} \mathrm{G}_{9 / 2}\right.$ ) (both spectral positions represented by dashed lines in Fig. 3) for several signal intensities $I_{0}$. Results are shown in Fig. 4.

Upper trace is a positive $\Delta I$ signal being responsible for SE: ${ }^{4} \mathrm{~F}_{3 / 2} \rightarrow{ }^{4} \mathrm{I}_{11 / 2}$, lower is a negative $\Delta I$ signal corresponding to the ESA: ${ }^{4} \mathrm{~F}_{3 / 2} \rightarrow{ }^{4} \mathrm{G}_{9 / 2}$. For small values of $I$ signal of stimulated emission increases with intensity of the probe beam, but after crossing some critical value it starts to decrease. The ESA signal grows with intensity of the probe beam in the entire range of the measurement. $\Delta I$ is a difference of the probe beam intensities when sample is pumped and unpumped, so the line on the top of Fig. 4 gives information of the dependence of light amplification on the intensity of the probe beam.

\section{Simulation}

We expect that the ESA transitions originating from ${ }^{4} \mathrm{~F}_{3 / 2}$ state diminishes the occupation of this state and amplification in the fiber goes down. A simple dependence between occupation of the excited state and amplification is the following:

$I=I_{0} e^{N_{e} \sigma_{\mathrm{SE}} l}$

where $I_{0}$ is the incident value of the probe beam intensity, $N_{e}$ is the population of the excited state, $\sigma_{\mathrm{SE}}$ is the stimulated emission cross section, and $l$ is the sample length. 


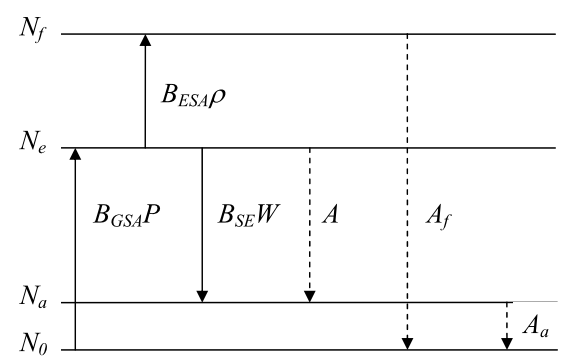

Fig. 5 Energy level diagram and transitions in $\mathrm{Nd}^{3+}$, used in simulation

If amplification decreases faster than $I_{0}$ increases, we can see a diminishing signal in spite of the increase of $I_{0}$. To check if ESA transitions are efficient enough to create such a situation we have attempted to simulate the occupation of states in $\mathrm{Nd}^{3+}$ and amplification of the fiber, using the simplest possible model, shown in Fig. 5.

The rate equations used in simulation are

$\dot{N}_{0}=-B_{\mathrm{GSA}} N_{0} P+A_{a} N_{a}+A_{f} N_{f}$,

$\dot{N}_{a}=A N_{e}+B_{\mathrm{SE}} N_{e} W-A_{a} N_{a}$,

$\dot{N}_{e}=-A N_{e}-B_{\mathrm{SE}} N_{e} W+B_{\mathrm{GSA}} N_{0} P-B_{\mathrm{ESA}} N_{e} \rho$,

$\dot{N}_{f}=B_{\mathrm{ESA}} N_{e} \rho-A_{f} N_{f}$,

$\dot{W}=B_{\mathrm{SE}} N_{e} W h v_{e}-W \kappa+A N_{e} h v_{e}$

where $N_{0}, N_{a}, N_{e}$ are occupations of states: ${ }^{4} \mathrm{I}_{9 / 2},{ }^{4} \mathrm{I}_{11 / 2}$, ${ }^{4} \mathrm{~F}_{3 / 2}$ and, respectively, all upper states are represented by $N_{f}, W$ is the density of stimulated radiation at $1097 \mathrm{~nm}$, originating from transition ${ }^{4} \mathrm{~F}_{3 / 2} \rightarrow{ }^{4} \mathrm{I}_{11 / 2}, \rho$ is the density of the probe beam radiation, $P$ is the pump beam radiation density, $B_{\mathrm{GSA}}, B_{\mathrm{ESA}}, B_{\mathrm{SE}}$ are the Einstein coefficients of the ground state absorption, excited state absorption and stimulated emission, respectively $A, A_{a}, A_{f}$ are spontaneous emission coefficients (as in Fig. 5) and $\kappa$ describes the photon losses in the fiber. In (3) the parameter $B_{\mathrm{ESA}} \rho$ is a sum of integrated Einstein coefficients multiplied by a suitable density value of the blackbody radiation, since a tungstenhalogen lamp serves as a source for the probe beam (see Fig. 6).

The expression relating $B_{\mathrm{ESA}} \rho$ and $\sigma_{\mathrm{ESA}}$ is then

$B_{\mathrm{ESA}} \rho=\sum_{i} \int \frac{c \sigma_{\mathrm{ESA}}^{i}(v)}{h v} \rho(v) d v$,

where $i$ enumerates the ESA transitions contributing to this calculations, which are ${ }^{4} \mathrm{~F}_{3 / 2} \rightarrow{ }^{4} \mathrm{G}_{5 / 2},{ }^{4} \mathrm{G}_{7 / 2},{ }^{4} \mathrm{G}_{9 / 2}$, ${ }^{2} \mathrm{~K}_{13 / 2}, \quad\left({ }^{2} \mathrm{G}(1),{ }^{4} \mathrm{G}_{9 / 2},{ }^{2} \mathrm{~K}_{15 / 2},{ }^{4} \mathrm{G}_{11 / 2},{ }^{4} \mathrm{D}_{3 / 2},{ }^{4} \mathrm{D}_{5 / 2}\right.$, ${ }^{4} \mathrm{D}_{7 / 2},{ }^{2} \mathrm{I}_{13 / 2}$.

The data used in calculations are as follows: $\sigma_{\mathrm{GSA}}=$ $4 \times 10^{-21} \mathrm{~cm}^{2}[14], \sigma_{\mathrm{SE}}=2.5 \times 10^{-20} \mathrm{~cm}^{2}[14], B_{\mathrm{ESA}} \rho=$ $64.6 \mathrm{~s}^{-1}\{$ taken from (4) $\}, A^{-1}=0.47 \times 10^{-3} \mathrm{~s}$ [15],

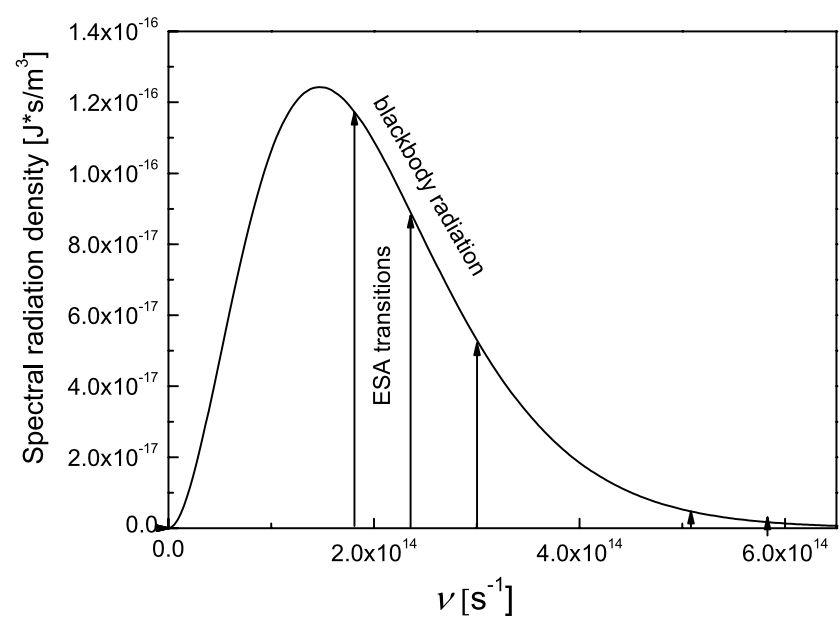

Fig. 6 ESA transitions situated on the blackbody radiation spectrum $(T=2500 \mathrm{~K})$

$A_{a}^{-1}=10^{-9} \mathrm{~s}[15], A_{f}^{-1}=10^{-4} \mathrm{~s}, \kappa=7 \times 10^{6} \mathrm{~s}^{-1}(\mathrm{de}-$ rived from geometry of the system), $\lambda_{\mathrm{GSA}}=514.5 \mathrm{~nm}$, $\lambda_{\mathrm{SE}}=1097 \mathrm{~nm}, N=2.1 \times 10^{19} \mathrm{~cm}^{-3}$ (concentration of $\mathrm{Nd}^{3+}$ ions). The sample was pumped by a $0.6 \mathrm{~W}$ laser beam.

In the experiment we used the pumping laser beam chopped with repetition of $5 \mathrm{~Hz}$. Because all considered transitions are much faster, we could solve the set of (3) as stationary equations by putting: $\dot{N}_{0}=\dot{N}_{a}=\dot{N}_{e}=\dot{N}_{f}=$ $\dot{W}=0$.

It is worthwhile to note that only the most efficient transitions are included in the model $[8,14]$. Our choice of the levels and transitions results from careful analysis of the JuddOfelt oscillator strengths and populations in $\mathrm{Nd}^{3+}$ ions in glass [14]. For example spontaneous transitions from the $N_{f}$ states to $N_{e}$ or $N_{a}$ are negligible because of small average occupation of the $N_{f}$ states. Hence the $A_{f}$ transitions are introduced mostly to obtain a closed set of equations and this set represents all the transitions from the $N_{f}$ states that feed, via different paths, the ground state $N_{0}$. We have checked numerically that taking into account a larger number of transitions makes no changes in the result of simulation, whereas it complicates the set of rate equations to a form which is analytically unsolvable. Because the used model is very simple the set of equations (3) can be solved analytically. Doing this we can find the dependence between excited state occupation and the probe beam intensity (or radiation density). The expression for $N_{e}(\rho)$ appears to be extremely complicated and too long to be shown but the reciprocal function is much simpler:

$\rho\left(N_{e}\right)=\frac{N-N_{e}+\frac{\kappa}{h v_{e}}\left(\frac{A}{B_{\mathrm{SE}}-\frac{\kappa}{N_{e} h v_{e}}}\right)\left(\frac{1}{A_{a}}+\frac{1}{B_{\mathrm{GSA}} P}\right)}{B_{\mathrm{ESA}} N_{e}\left(\frac{1}{A_{f}}+\frac{1}{B_{\mathrm{GSA}} P}\right)}$.

Apart from the analytical solution (5) we have solved the set (3) numerically and both solutions were identical. In case 


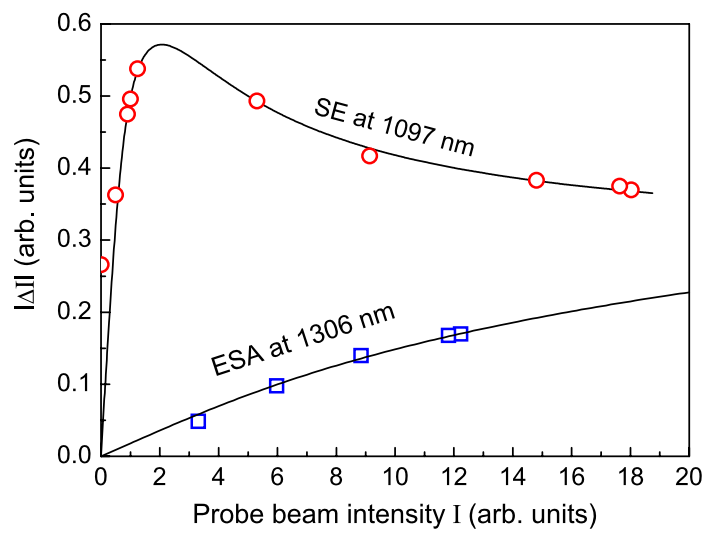

Fig. 7 Comparison of experimental data and calculations for $\Delta I$ dependence on the probe beam intensity

of numerical solution we simulated different probe beam intensities by altering the parameter $B_{\mathrm{ESA}} \rho$ and solving the set of equations for its fixed values. Having values of $N_{e}$ we calculated amplification and difference between radiation intensity in presence and in absence of the pump beam:

$\Delta I=I_{0} e^{N_{e} \sigma_{\mathrm{SE}} l}-I_{0}$.

In this way we have obtained theoretical dependence on the top of Fig. 7, which fits the stimulated emission. Signal of the ESA transition ${ }^{4} \mathrm{~F}_{3 / 2} \rightarrow{ }^{4} \mathrm{G}_{9 / 2}$ is shown below and it is proportional to

$\Delta I=I_{0} e^{N_{e} \sigma_{\mathrm{ESA}} l}-I_{0}$.

The parameter used to fit the theoretical dependencies to the experimental data was the initial value of the product $N_{e} l$ because we should use an effective optical length instead of the fiber length when the concentration of excited $\mathrm{Nd}^{3+}$ ions is not homogeneous. As we can see, calculations fit well to the experimental results. When we increase the intensity of the probe beam, the number of the ESA transition acts increases and occupation of the excited state decreases. For small $I_{0}$ values the signal of stimulated emission grows, because the amplification is larger than the ESA effects. When the ESA transitions are very efficient, because of the high probe beam intensity, amplification decreases faster than the increase of the input intensity and, as a result, the output gain decreases. It is possible when the exponential factor in (6) decreases faster than $I_{0}$ increases. $\Delta I$ signal at $1306 \mathrm{~nm}$ (ESA: ${ }^{4} \mathrm{~F}_{3 / 2} \rightarrow{ }^{4} \mathrm{G}_{9 / 2}$ ) grows with $I_{0}$ because this ESA cross section is here about three times smaller than the SE cross section (for ${ }^{4} \mathrm{~F}_{3 / 2} \rightarrow{ }^{4} \mathrm{I}_{11 / 2}$ ) and then the exponential factor in (7) decreases smaller than $I_{0}$ increases. The dependence between $\Delta I$ signal, amplification and probe beam intensity is shown in Fig. 8. If the exponential factor in (6) or (7) is small, because of small value of $\mathrm{Nl}$ or $\sigma$, the signal $\Delta I$ will grow with $I_{0}$, like the first five lines in Fig. 8, but for large

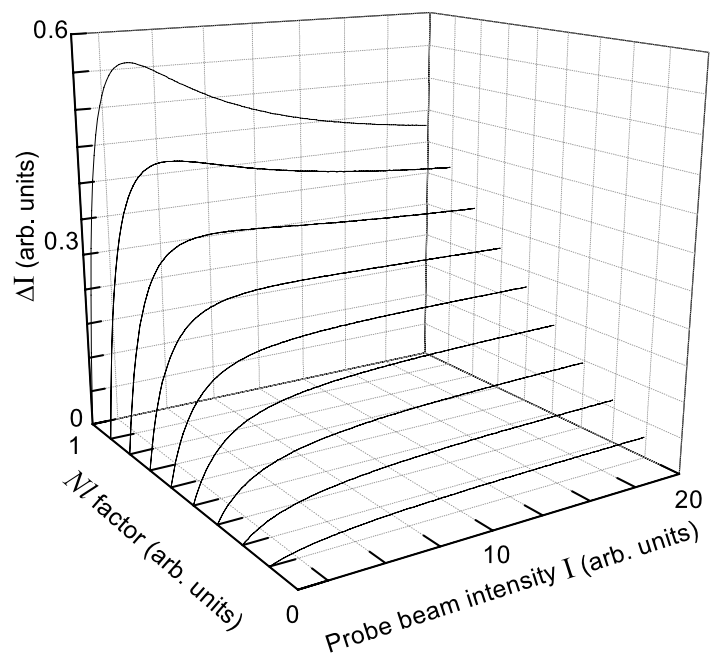

Fig. 8 Comparison of $\Delta I$ spectra for different $N l$ factors

values of these parameters $\Delta I$ will change with $I_{0}$ like the last four lines. The last line in Fig. 8 is the result of calculations for the SE transition $\left({ }^{4} \mathrm{~F}_{3 / 2} \rightarrow{ }^{4} \mathrm{I}_{11 / 2}\right)$ with parameters characterized our experiment, so if the ESA cross section $\left({ }^{4} \mathrm{~F}_{3 / 2} \rightarrow{ }^{4} \mathrm{G}_{9 / 2}\right)$ is three times smaller than the SE cross section, the third line should fit to the $\Delta I$ signal at $1306 \mathrm{~nm}$. This is also a reason why we cannot observe such strange $\Delta I$ vs. $I_{0}$ dependence in bulks, where the exponential factor is small because of small probe length.

\section{Conclusions}

Designing broadband amplifiers we should take the ESA transitions into account. Using higher intensities of the input signal one will see amplification much smaller than expected, and in extreme case no amplification will be observed. The ESA transitions are efficient enough to decrease amplification and make the competition between input and output signals i.e. output is falling when input intensity is growing. Moreover, as presented in Fig. 8, the influence of the ESA on the gain depends on the fiber length. The mentioned nonmonotonic relation between the gain and signal intensity occurs for suitably long fibers. This competition exists despite of the fact that the gain and the ESA features occur in different spectral ranges. Registering the ESA spectra in the fibers one should be careful because using different intensities of the probe beam we can obtain different spectra. On other hand it is possible to use this effect to separate the ESA from the SE transitions occurring at the same wavelengths and originating from the same state. One should not find this effect in bulks because their amplification is much smaller for the same dopant concentration and any changes in gain would be negligible. On the other hand, for the bulk material of the dopant concentration high enough to produce 
the described effect one should expect the influence of the ion-ion energy transfer which would entirely change the system behavior.

In this context it is perhaps important to note that our results follow exclusively from the length of the active medium. This is why the optical fiber reveals the described phenomena (we do not take into account any other parameters of the fibers, e.g., depending on the fiber cross section, the only important parameter is its length) as far as is activated by relatively small concentration of dopant. In other words: a $9 \mathrm{~m} \times 9 \mathrm{~m} \times 9 \mathrm{~m}$ huge sample of the same medium (and with the same dopant concentration) should also reveal the phenomena described.

Acknowledgements We are very grateful to Dr. J. Wójcik of the Department of Optical Fibers Technology, University of Maria CurieSkłodowska, Lublin, Poland, for providing the samples and to Dr. B. Ziętek of our Institute for fruitful discussions. The work was supported by the local grant of the Nicolaus Copernicus University (under number 373-F) and by the European Social Fund and the state budget under the project "Scholarship for PhD Students 2008/2009-ZPORR" of the Regional Council of Kujawsko-Pomorskie Province.

Open Access This article is distributed under the terms of the Creative Commons Attribution Noncommercial License which permits any noncommercial use, distribution, and reproduction in any medium, provided the original author(s) and source are credited.

\section{References}

1. M.J.F. Digonnet (ed.), Rare-Earth-Doped Fiber Lasers and Amplifiers, 2nd edn. (CRC Press/Taylor \& Francis, Boca Raton, 2001)

2. H. Shalibeik, Rare-Earth-Doped Fiber Lasers and Amplifiers (Cuvillier, Göttingen, 2007)

3. J. Ruan, E. Wu, H. Zeng, S. Zhou, G. Lakshminarayana, J. Qiu, Appl. Phys. Lett. 92, 101121 (2008)

4. X. Wang, P. Zhou, Y. Ma, H. Ma, X. Xu, Z. Liu, Y. Zhao, J. Opt. 12, $075701(2010)$

5. T. Sakamoto, M. Shimizu, M. Yamada, T. Kanamori, Y. Ohishi, Y. Terunuma, S. Sudo, IEEE Photonics Technol. Lett. 8, 349 (1996)

6. S.A. Zemon, G.M. Lambert, W.J. Miniscalco, R.W. Davies, B.T. Hall, R.C. Folweiler, Wei Ta-Sheng, L.J. Andrews, M.P. Singh, Proc. SPIE 1373, 21 (1991). doi:10.1117/12.24926

7. D. Piątkowski, K. Wiśniewski, M. Różański, Cz. Koepke, M. Kaczkan, M. Klimczak, R. Piramidowicz, M. Malinowski, J. Phys., Condens. Matter 20, 155201 (2008)

8. J.L. Adam, J.L. Doualan, L. Griscom, S. Girard, R. Moncorgé, J. Non-Cryst. Solids 256, 276 (1999)

9. Cz. Koepke, Opt. Mater. 30, 674 (2008)

10. D. Piątkowski, K. Wiśniewski, C. Koepke, R. Piramidowicz, M. Klimczak, M. Malinowski, Appl. Phys. B 93, 809 (2008)

11. A. Ghatak, K. Thyagarajan, Introduction to Fiber Optics (Cambridge University Press, Cambridge, 1998)

12. C. Barnard, P. Myslinski, J. Chrostowski, M. Kavehrad, IEEE J. Quantum Electron. 30, 1817 (1994)

13. D.F. de Sousa, V. Peters, G. Huber, A. Toncelli, D. Parisi, M. Tonelli, Appl. Phys. B 77, 817 (2003)

14. D. Piątkowski, K. Wiśniewski, Cz. Koepke, M. Naftaly, Opt. Mater. 31, 541 (2009)

15. M. Malinowski, Lasery świattowodowe (Oficyna Wydawnicza Politechniki Warszawskiej, Warszawa, 2003), p. 87 